\title{
Prognostic Value of Tissue Inhibitor of Metalloproteinase in Acute Aortic Dissection
}

\author{
Naohiko Inagaki ${ }^{*},{ }$, Kimihiko Kato ${ }^{2}$, Toshiki Kawamiya ${ }^{1}$, Hideki Horibe ${ }^{1}$, Takeshi Hibino ${ }^{1}$, \\ Kiyoshi Yokoi ${ }^{1}$, Yasuaki Dohi ${ }^{3}$, Toyoaki Murohara ${ }^{4}$ and Genjiro Kimura ${ }^{3}$ \\ ${ }^{I}$ Department of Cardiovascular Medicine, Gifu Prefectural Tajimi Hospital, Tajimi, Japan \\ ${ }^{2}$ Department of Internal Medicine, Meito Hospital, Nagoya, Japan \\ ${ }^{3}$ Department of Cardio-Renal Medicine and Hypertension, Nagoya City University Graduate School of Medical \\ Sciences, Nagoya, Japan \\ ${ }^{4}$ Department of Cardiology, Nagoya University Graduate School of Medicine, Nagoya, Japan
}

\begin{abstract}
Background: Increases in levels of matrix metalloproteinase (MMP) and tissue inhibitor of matrix metalloproteinase (TIMP) occur in acute aortic dissection (AAD); however, their association with prognosis in AAD remains largely unknown. We studied the use of MMP and TIMP in predicting long-term outcomes in medically controlled AAD patients.

Methods: A total of 82 patients were enrolled (15 Stanford Type A and 67 type B, age $65.1 \pm 13.3$ ). AAD was diagnosed by enhanced CT, with serial imaging studies during follow-up. Blood tests for MMP-2, MMP-9, TIMP-1, and TIMP-2 were performed within a week after symptom onset.

Results: A poor outcome due to death or aortic surgical repair occurred in 17 patients, classified as an unfavorable group. The remaining 65 patients were classified into a favorable group. By multivariate analysis maximum dissection diameter (MDD), TIMP-1, and TIMP-2 were significantly associated with an unfavorable outcome $(\mathrm{P}<0.001, \mathrm{P}<0.001$, and $\mathrm{P}=$ 0.038 , respectively). The ratio of TIMP-1 to TIMP-2 (cut-off value $>3.34$ ) had significant predictive power (sensitivity $88.2 \%$, specificity $80.0 \%$ ). Patients with MDD $\geq 46.8 \mathrm{~mm}$ and a TIMP-1/TIMP-2 ratio $\geq 3.34$ had a significantly poorer outcome (log rank $\mathrm{P}<0.0001)$. Similarly, in Cox regression analysis AAD patients with MDD $\geq 46.8 \mathrm{~mm}$ and a TIMP1/TIMP-2 ratio $\geq 3.34$ had the highest risk for an unfavorable outcome $(\mathrm{P}<0.001$, hazard ratio $45.6,95 \%$ confidence interval 5.96 to 348.42 ).

Conclusion: In AAD a higher TIMP-1/TIMP-2 ratio was significantly associated with an increased risk of death or surgical repair.
\end{abstract}

Keywords: Aortic dissection, risk factors, follow-up studies, matrix metalloproteinase, tissue inhibitor of matrix metalloproteinase.

\section{INTRODUCTION}

Acute aortic dissection (AAD) is a life-threatening condition, and is a leading cause of mortality [1] that arises from an atherosclerotic lesion in the aorta. Current outcomes of medical therapy for Stanford type B patients with AAD remain high, with early mortality ranging from 10 to $12 \%$ [2, 3]. Medical management for AAD, particularly for Stanford type B and type A intramural hematoma, poses a difficult problem in deciding on surgery, and when the procedure should be performed [4-6]. The decision should balance the surgical risk and the hazard of aortic rupture in elective cases of Stanford type B, type A intramural hematoma, and also in the elderly [4-7]. Close long-term monitoring of morphologic parameters including maximum aortic diameter and the configuration of dissection by computed tomography (CT) is helpful for both prevention of aortic rupture, and for

*Address correspondence to this author at the Department of Cardiovascular Medicine, Gifu Prefectural Tajimi Hospital, 5-161 Maehata, Tajimi, Gifu 5078522, Japan; Tel: +81 57222 5311; Fax: +81 57225 1246;

E-mail: inagaki-naohiko@tajimi-hospital.jp undertaking timely surgical or endovascular interventions [8]. However, only the relative and not the individual rupture risk, can be determined [9]. Hence, a better understanding is sought for the factors relevant to the progressive need for surgical intervention and rupture of AAD during conservative medical therapy. Such identifying factors may, in turn, lead to better selection of AAD patients with higher risk of rupture requiring conversion to surgical therapy.

Degenerative and dissecting aneurysms of the aorta develop as a result of progressive weakening of the aortic wall. Some current studies have demonstrated that metalloproteinases (MMP) and tissue inhibitor of metalloproteinase (TIMP) are related to tissue destruction and repair $[10,11]$, and increased levels of MMP and TIMP have been observed in AAD patients [12]. However, the presence or absence of an association between levels of MMP, TIMP and the prognosis in AAD remains largely unknown.

This study aims to explore the clinical utility of MMPs and TIMPs for the prediction of long-term outcomes in medically treated patients with AAD. 


\section{MATERIALS AND METHODS}

\section{Patient Population and Study Protocol}

A retrospective study was conducted in which unfavorable and favorable outcomes in AAD patients were compared. There were 101 patients with AAD who had emergency admissions to our hospital between September, 2003 and May, 2009. Of this number 11 patients died and 8 patients underwent surgical repair during the acute phase of hospitalization. The remaining 82 patients (56 males and 26 females, aged $65.1 \pm 13.3$ ) were enrolled in this study.

All patients were initially treated medically, consisting of those with Stanford type B $(n=67)$, as well as those with Stanford type A $(n=15)$ in whom surgery was felt to be contraindicated due to intramural hematoma, severe comorbidity, or advanced age. All patients were examined initially by chest $\mathrm{x}$-ray, laboratory tests for D-dimer, electrocardiograms, and echocardiography followed by contrast medium-enhanced CT. Aortic dissection was defined as a separation of the aortic wall layers, with resulting true and false lumens, or as intramural hematoma determined by enhanced chest CT [7]. Individuals with chronic renal disease (serum creatinine level $>2.0 \mathrm{mg} / \mathrm{dl}$ ), Marfan syndrome, Ehlers-Danlos syndrome, traumatic aneurysm, bicuspid aortic valve, arteritis, pseudoaneurysm, mycotic aneurysm, connective tissue disorder, congenital malformations of the heart or vessels, and patients who had experienced from death or surgical repair of aorta during the initial hospitalization were excluded from the study.

Blood samples were drawn for lipid profiles, glycosylated hemoglobin $\mathrm{A}_{1 \mathrm{c}}$, D-dimer, serum MMP-2, serum MMP-9, serum TIMP-1 and serum TIMP-2. D-dimer was measured by latex agglutination. The value of MMP-2, MMP-9, TIMP-1, and TIMP-2 was evaluated using the enzyme-linked immunosorbent assay kit (DAIICHI FINECHEMICAL, JAPAN). We added $50 \mu \mathrm{L}, 50 \mu \mathrm{L}, 10$ $\mu \mathrm{L}$, and $100 \mu \mathrm{L}$, respectively of each of the standard recombinant human MMP-2, MMP-9, TIMP-1, and TIMP-2. The supernatant extracted from the sample was also added. Also added was $300 \mu \mathrm{L}$ of horseradish peroxidase (HRP) labeled anti-MMP-2, -MMP-9, -TIMP-2 to an antihuman antibody-solidified microtiter plate. $150 \mu \mathrm{L}$ of HRP labeled anti-TIMP-1 was added to an antihuman antibody-solidified microtiter plate. MMP-2, MMP-9, and TIMP-2 were incubated at room temperature for $1 \mathrm{~h}$; TIMP-1 for $30 \mathrm{~min}$. The residue was washed 3 times in each case. $300 \mu \mathrm{L}$ of OPhenylendediamine was added and reacted at room temperature for $30 \mathrm{~min}$ for MMP-2 and MMP-9; $100 \mu \mathrm{L}$ and $15 \mathrm{~min}$ for TIMP-1; and $300 \mu \mathrm{L}$ of $3,3^{\prime}, 5,5^{\prime}-$ tertamethylbenzidine and $30 \mathrm{~min}$ for TIMP-2. Finally, $1 \mathrm{M}$ sulfuric acid was added to stop the reaction; the absorbance was measured with a microplate reader (Molecular Devices, Tokyo, Japan) at a wavelength of $492 / 630 \mathrm{~nm}$, and the concentration was analyzed from the standard curve for MMP-2, MMP-9, and TIMP-1; 450/630 nm for TIMP-2.

The definition of an unfavorable outcome included death due to aortic rupture, and ultimate requirement for surgical repair after discharge of the initial hospitalization. All patients received anti-hypertensive therapy according to the usual standard of care, and were followed up with a physician's examination and enhanced chest CT. The study protocol complied with the Declaration of Helsinki, and was approved by the Committee on the Ethics of Human Research of Gifu Prefectural Tajimi Hospital. Each patient gave written informed consent.

\section{Data Analysis}

Data analysis was carried out using the statistical package SPSS version 11.01J for windows (SPSS Japan, Inc., Tokyo, Japan). Continuous variables were expressed as mean $\pm \mathrm{SD}$, unless otherwise stated, and were compared using the Mann-Whitney test if data did not show normal distribution. Student's t test was used to compare groups characterized by continuous variables with normal distribution. Categorical variables, expressed as percentages, were compared by the chi-square test or Fisher's exact test. Significant univariate risk factors were followed by application of multivariate analysis using conventional logistic regression. Hazard ratios were calculated using Cox regression analysis. Receiver operating characteristic (ROC) curves were constructed to assess predictive power, with calculation of the area under the curve (AUC). Statistical significance was examined by two-sided tests. Survival curves of patient groups were calculated by the KaplanMeier method and compared with the log rank test. P values $<0.05$ were considered significant.

\section{RESULTS}

The mean observation period was $18.8 \pm 15.1$ months. During the initial hospitalization life-threatening events such as stroke $(\mathrm{n}=1)$, cardiac tamponade $(\mathrm{n}=7)$, pleural effusion $(\mathrm{n}=21)$, shock $(\mathrm{n}=3)$, acute renal failure $(\mathrm{n}=13)$, mesenteric ischemia $(n=5)$, and limb ischemia $(n=6)$ were observed as complications accompanying AAD. Some of these life-threatening events were associated with further complications, and ultimately contributed to the outcome of late stage death $(n=7)$ or surgical repair $(n=10)$ among the 17 patients classified into the unfavorable AAD group. The remaining 65 patients were classified into the favorable AAD group.

Table 1 shows baseline characteristics of the study subjects. Stanford type A, presence of hypotension and shock, and presence of aortic valve regurgitation were all significantly more common in the unfavorable than in the favorable AAD group. No adverse events related to medication occurred during the hospitalization.

Table 2 shows data from CT and levels of measured biomarkers in the two groups. Maximum dissection diameter, and mean values of serum MMP-2 and serum TIMP-1 were significantly greater in the unfavorable than in the favorable AAD group, and values of serum TIMP-2 were significantly lower. And Table 2 demonstrated that MMP2/TIMP-2 ratio and TIMP-1/TIMP-2 ratio were significantly difference in the two group $(\mathrm{P}=0.003$ and $\mathrm{P}<0.001$, respectively).

Table 3 presents data for multivariate predictors of baseline characteristics, data from chest $\mathrm{CT}$, and laboratory tests in patients with AAD. In multivariate analysis using a conventional logistic regression model, maximum dissection diameter and mean value of serum TIMP-1 were independently and positively associated with death or surgical repair $(\mathrm{P}=0.008$, odds ratio, $1.18,95 \%$ confidence 
Table 1. Baseline Characteristics of the Study Subjects

\begin{tabular}{|c|c|c|c|c|}
\hline \multirow{2}{*}{ Characteristic } & \multirow{2}{*}{ Test } & Favorable AAD & Unfavorable AAD & \multirow{2}{*}{$\mathbf{P}$} \\
\hline & & $(n=65)$ & $(\mathbf{n}=17)$ & \\
\hline Age (years) & $\mathrm{T}$ & $64.2 \pm 13.2$ & $68.7 \pm 13.3$ & 0.215 \\
\hline Female $(\%)$ & $\mathrm{X}$ & $21(32.3)$ & $5(29.4)$ & 0.819 \\
\hline Body mass index $\left(\mathrm{kg} / \mathrm{m}^{2}\right)$ & $\mathrm{M}-\mathrm{W}$ & $24.0 \pm 4.4$ & $22.3 \pm 1.4$ & 0.147 \\
\hline Stanford A (\%) & $\mathrm{X}$ & $8(12.3)$ & $7(41.2)$ & 0.006 \\
\hline \multicolumn{5}{|l|}{ Past medical history } \\
\hline Hypertension (\%) & $\mathrm{X}$ & $56(86.2)$ & $15(88.2)$ & 0.823 \\
\hline Hyperlipidemia (\%) & $\mathrm{X}$ & $16(24.6)$ & $3(17.6)$ & 0.544 \\
\hline Diabetes mellitus (\%) & $\mathrm{X}$ & $6(9.2)$ & $2(11.8)$ & 0.754 \\
\hline Coronary artery disease (\%) & $\mathrm{X}$ & $3(4.6)$ & $1(5.9)$ & 0.829 \\
\hline Prior cardiac surgery (\%) & $\mathrm{X}$ & $2(3.1)$ & $1(5.9)$ & 0.583 \\
\hline COPD (\%) & $\mathrm{X}$ & $17(26.2)$ & $8(47.1)$ & 0.096 \\
\hline \multicolumn{5}{|l|}{ Clinical presentation } \\
\hline Chest and back pain (\%) & $\mathrm{X}$ & $55(84.6)$ & $12(70.6)$ & 0.163 \\
\hline Migrating pain (\%) & $\mathrm{X}$ & $21(32.3)$ & $7(41.2)$ & 0.547 \\
\hline Radiating pain (\%) & $\mathrm{X}$ & $5(7.7)$ & $3(17.6)$ & 0.218 \\
\hline Any pulse deficit (\%) & $\mathrm{X}$ & $3(4.6)$ & $0(0)$ & 0.367 \\
\hline Neurological deficit (\%) & $\mathrm{X}$ & $2(3.1)$ & $0(0)$ & 0.464 \\
\hline Hypotension and shock (\%) & $\mathrm{X}$ & $1(1.5)$ & $2(11.8)$ & 0.046 \\
\hline \multicolumn{5}{|l|}{ Complication } \\
\hline Cardiac tamponade (\%) & $\mathrm{X}$ & $6(9.2)$ & $1(5.9)$ & 0.660 \\
\hline Aortic valve regurgitation (\%) & $\mathrm{X}$ & $8(12.3)$ & $6(35.3)$ & 0.025 \\
\hline Congestive heart failure (\%) & $\mathrm{X}$ & $4(6.2)$ & $2(11.8)$ & 0.429 \\
\hline Stroke (\%) & $\mathrm{X}$ & $1(1.5)$ & $0(0)$ & 0.607 \\
\hline Acute renal failure (\%) & $\mathrm{X}$ & $8(12.3)$ & $5(29.4)$ & 0.086 \\
\hline Mesenteric ischemia (\%) & $\mathrm{X}$ & $4(6.2)$ & $1(5.9)$ & 0.967 \\
\hline Limb ischemia (\%) & $\mathrm{X}$ & $5(7.7)$ & $1(5.9)$ & 0.799 \\
\hline \multicolumn{5}{|l|}{ Chest X-ray } \\
\hline Abnormal cardiac contour (\%) & $\mathrm{X}$ & $43(66.2)$ & $14(82.4)$ & 0.196 \\
\hline Widened mediastinum (\%) & $\mathrm{X}$ & $47(72.3)$ & $13(76.5)$ & 0.730 \\
\hline Pleural effusion (\%) & $\mathrm{X}$ & $15(23.1)$ & $6(35.3)$ & 0.304 \\
\hline \multicolumn{5}{|l|}{ ECG abnormalities } \\
\hline Left ventricular hypertrophy (\%) & $\mathrm{X}$ & $16(24.6)$ & $4(23.5)$ & 0.926 \\
\hline ST elevation (\%) & $\mathrm{X}$ & $12(18.5)$ & $5(29.4)$ & 0.321 \\
\hline ST depression (\%) & $\mathrm{X}$ & $13(20.0)$ & $6(35.3)$ & 0.183 \\
\hline Abnormal Q wave (\%) & $\mathrm{X}$ & $7(10.8)$ & $1(5.9)$ & 0.545 \\
\hline Initial medication (\%) & $\mathrm{X}$ & & & 0.063 \\
\hline Beta-blocker & & $55(84.6)$ & $12(70.6)$ & \\
\hline Calcium channel blocker & & $50(76.9)$ & $9(52.9)$ & \\
\hline ACE inhibitor & & $7(10.8)$ & $3(17.6)$ & \\
\hline ARB & & $51(78.5)$ & $7(41.2)$ & \\
\hline Statins & & $23(35.4)$ & $4(23.5)$ & \\
\hline
\end{tabular}

AAD, acute aortic dissection; ACE, angiotensin converting enzyme; ARB, angiotensin receptor blocker; COPD, chronic obstructive pulmonary disease; ECG, electrocardiogram Abbreviations for statistical tests: M-W, Mann-Whitney test; T, Student's $t$ test; $\mathbf{X}$, chi-square test or Fisher's exact test. Data are presented as No. of patients (\%) or mean \pm SD unless otherwise indicated. P values are for the differences between favorable and unfavorable AAD groups, and significant $\mathrm{P}$ values $(<0.05)$ are expressed in bold face.

interval 1.04-1.33; $\mathrm{P}=0.008$, odds ratio $1.02,95 \%$ confidence interval 1.01-1.04, respectively), whereas serum TIMP-2 was negatively associated $(\mathrm{P}=0.038$, odds ratio $0.95,95 \%$ confidence interval 0.91-1.00). Additionally, the ratio between MMPs and TIMPs was calculated. The ratio for TIMP-1/TIMP-2 and MMP-2/TIMP-2 in the unfavorable AAD group was significantly higher than in the favorable AAD group $(\mathrm{P}=0.008$, odds ratio $2.00,95 \%$ confidence interval 1.20-3.33; $\mathrm{P}=0.011$, odds ratio $1.26,95 \%$ confidence interval 1.06-1.15, respectively). 
Table 2. Data from Chest CT and Laboratory Tests in Patients with AAD

\begin{tabular}{|c|c|c|c|c|}
\hline \multirow{2}{*}{ Characteristic } & \multirow{2}{*}{ Test } & Favorable AAD & Unfavorable AAD & \multirow{2}{*}{$\mathbf{P}$} \\
\hline & & $(n=65)$ & $(\mathbf{n}=17)$ & \\
\hline \multicolumn{5}{|l|}{ Chest CT findings } \\
\hline Intramural hematoma (\%) & $\mathrm{X}$ & $35(53.8)$ & $6(35.3)$ & 0.173 \\
\hline True lumen compression (\%) & $\mathrm{X}$ & $19(29.2)$ & $7(41.2)$ & 0.346 \\
\hline Thrombosed false lumen (\%) & $\mathrm{X}$ & $50(76.9)$ & $12(70.6)$ & 0.588 \\
\hline False to true lumen ratio & $\mathrm{T}$ & $0.8 \pm 0.8$ & $1.1 \pm 1.1$ & 0.251 \\
\hline Maximum dissection diameter $(\mathrm{mm})$ & $\mathrm{T}$ & $40.7 \pm 8.0$ & $52.7 \pm 10.7$ & $<0.001$ \\
\hline \multicolumn{5}{|l|}{ Laboratory tests } \\
\hline LDL cholesterol (mg/dl) & $\mathrm{T}$ & $118.3 \pm 34.3$ & $107.0 \pm 35.3$ & 0.264 \\
\hline HDL cholesterol (mg/dl) & $\mathrm{T}$ & $46.7 \pm 13.3$ & $40.2 \pm 15.0$ & 0.639 \\
\hline Triglyceride (mg/dl) & $\mathrm{T}$ & $105.8 \pm 60.0$ & $107.1 \pm 78.6$ & 0.945 \\
\hline $\mathrm{HBA}_{\mathrm{lc}}(\%)$ & $\mathrm{T}$ & $6.0 \pm 4.8$ & $5.5 \pm 0.4$ & 0.641 \\
\hline Serum creatinine level (mg/dl) & $\mathrm{T}$ & $0.8 \pm 0.5$ & $0.9 \pm 0.4$ & 0.639 \\
\hline D-dimer $(\mu \mathrm{g} / \mathrm{ml})$ & $\mathrm{T}$ & $76.3 \pm 283.7$ & $84.0 \pm 169.4$ & 0.918 \\
\hline MMP-2 (ng/ml) & $\mathrm{T}$ & $633.2 \pm 152.2$ & $749.5 \pm 227.5$ & 0.014 \\
\hline MMP-9 (ng/ml) & $\mathrm{T}$ & $88.5 \pm 83.4$ & $87.2 \pm 95.3$ & 0.957 \\
\hline TIMP-1 (ng/ml) & M-W & $182.4 \pm 49.9$ & $245.9 \pm 75.4$ & 0.001 \\
\hline TIMP-2 (ng/ml) & $\mathrm{T}$ & $69.5 \pm 26.5$ & $55.8 \pm 15.7$ & 0.046 \\
\hline MMP-2 / MMP-9 & $\mathrm{T}$ & $14.6 \pm 13.8$ & $21.2 \pm 19.5$ & 0.114 \\
\hline MMP-2 / TIMP-1 & $\mathrm{T}$ & $3.7 \pm 1.2$ & $3.3 \pm 1.3$ & 0.305 \\
\hline MMP-2 / TIMP-2 & $\mathrm{T}$ & $10.0 \pm 3.8$ & $14.8 \pm 7.7$ & 0.003 \\
\hline MMP-9 / TIMP-1 & $\mathrm{T}$ & $0.5 \pm 0.5$ & $0.3 \pm 0.4$ & 0.225 \\
\hline MMP-9 / TIMP-2 & $\mathrm{T}$ & $1.2 \pm 0.9$ & $1.7 \pm 2.0$ & 0.668 \\
\hline TIMP-1 / TIMP-2 & $\mathrm{T}$ & $2.9 \pm 1.2$ & $4.7 \pm 2.3$ & $<0.001$ \\
\hline
\end{tabular}

AAD, acute aortic dissection; CT, computed tomography; $\mathbf{H B A}_{\mathbf{l c}}$, glycosylated hemoglobin $\mathrm{A}_{1 \mathrm{c}} ; \mathbf{H D L}$, high density lipoprotein; LDL, low density lipoprotein; MMP, matrix metalloproteinase; TIMP, tissue inhibitor of metalloproteinase. Abbreviations for statistical tests: M-W, Mann-Whitney test; T, Student's t test; X, chi-square test or Fisher's exact test. Data are presented as number of patients (\%) or mean \pm SD unless otherwise indicated. P values are for the differences between favorable and unfavorable AAD groups, and significant $\mathrm{P}$ values $(<0.05)$ are expressed in bold face.

Analysis of the AUC assessed the predictive accuracy of maximum dissection diameter, TIMP-1/TIMP-2 ratio and MMP-2/TIMP-2 ratio for long-term outcomes of AAD (Fig. 1). The AUC for maximum dissection diameter was $0.842 \pm 0.052$ (mean \pm standard error of mean), and the AUC for TIMP-1/TIMP-2 ratio was $0.833 \pm 0.056$, both approximately the same in magnitude. Meanwhile, the AUC for MMP-2/TIMP-2 ratio was $0.733 \pm 0.074$. The AUC for MMP-2/TIMP-2 ratio was smaller than the AUC for TIMP1/TIMP-2 ratio. For the prediction of an unfavorable outcome the optimal cut-off level of maximum dissection diameter determined from the ROC curves was $46.8 \mathrm{~mm}$, which had the following predictive powers: sensitivity $88.2 \%$, specificity, $80.0 \%$, positive predictive value $53.6 \%$, negative predictive value $96.3 \%$, accuracy $81.7 \%$, odds ratio 30.0 .

The optimal cut-off level of the TIMP-1/TIMP-2 ratio determined from the ROC curves was 3.34, which demonstrated the following predictive powers: sensitivity $88.2 \%$, specificity $80.0 \%$, positive predictive value $53.6 \%$, negative predictive value $96.3 \%$, accuracy $81.7 \%$, odds ratio, 30.0. Stratification of patients according to maximum aortic dissection diameter $\geq 46.8 \mathrm{~mm}$ and TIMP-1/TIMP-2 ratio $\geq$ 3.34 gave additional information on outcomes (Fig. 2). Patients could can be classified as having low risk (both markers lower than cut-off values), intermediate risk (either marker greater than the cut-off value), or high risk (both markers greater than cut-off values; log rank for trend $\mathrm{P}<$ $0.0001)$.

Table 4 shows the results of univariate Cox regression analysis for prediction of death or surgical repair in patients stratified according to a maximum aortic dissection diameter $(<$ or $\geq 46.8 \mathrm{~mm})$ in relation to TIMP-1/TIMP-2 ratios $(<$ or $\geq 3.34)$. AAD patients with greater $(\geq 46.8 \mathrm{~mm}$ ) maximum aortic dissection diameters and a higher $(\geq 3.34)$ TIMP1/TIMP-2 ratio had the highest risk for death or surgical repair compared with patients having a smaller $(<46.8 \mathrm{~mm})$ maximum aortic dissection diameter and a lower $(<3.34)$ TIMP-1/TIMP-2 ratio $(\mathrm{P}<0.001$, hazard ratio $45.6,95 \%$ confidence interval 5.96-348.42). 
Table 3. Multivariate Predictors of Baseline Characteristics, Data from Chest CT and Laboratory Tests in Patients with AAD

\begin{tabular}{|l|l|c|}
\hline \multicolumn{1}{|c|}{ Variable } & OR (95\% CI) & p \\
\hline \hline Stanford A & & 0.756 \\
\hline Clinical presentation & & \\
\hline Hypotension and shock & & 0.312 \\
\hline Complication & & \\
\hline Aortic valve regurgitation & & 0.961 \\
\hline Chest CT findings & & \\
\hline Maximum dissection diameter $(\mathrm{mm})$ & $1.18(1.04-1.33)$ & $\mathbf{0 . 0 0 8}$ \\
\hline Laboratory tests & & \\
\hline MMP-2 (ng/ml) & & 0.064 \\
\hline TIMP-1 (ng/ml) & $1.02(1.01-1.04)$ & $\mathbf{0 . 0 0 8}$ \\
\hline TIMP-2(ng/ml) & $0.95(0.91-1.00)$ & $\mathbf{0 . 0 3 8}$ \\
\hline MMP-2/TIMP-2 ratio & $1.26(1.06-1.51)$ & $\mathbf{0 . 0 1 1}$ \\
\hline TIMP-1/TIMP-2 ratio & $2.00(1.20-3.33)$ & $\mathbf{0 . 0 0 8}$ \\
\hline
\end{tabular}

AAD, acute aortic dissection; CI, confidence interval; CT, computed tomography; MMP, matrix metalloproteinase; OR, odds ratio; TIMP, tissue inhibitor of metalloproteinase: Data are presented as mean \pm SD unless otherwise indicated. Significant $\mathrm{P}$ values $(<0.05)$ by analysis of variance followed by a multiple comparison test are expressed in bold face.

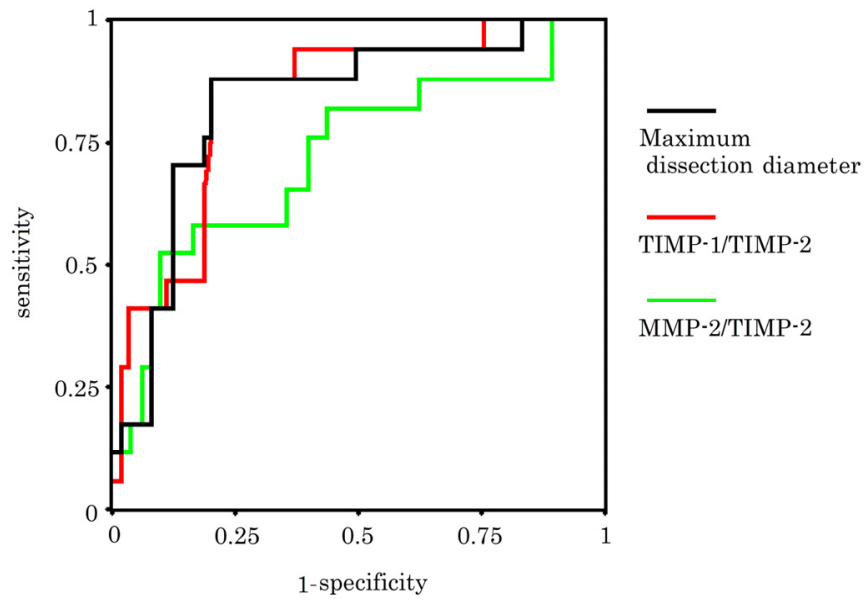

Fig. (1). ROC curves - ROC curves of TIMP-1/TIMP-2 ratio and maximum dissection diameter for discriminating AAD patients with favorable outcome $(\mathrm{n}=65)$ vs unfavorable outcome $(\mathrm{n}=17)$. The area under receiver operating characteristic curves for TIMP1/TIMP-2 ratio was 0.833 and for maximum dissection diameter was 0.842: AAD, acute aortic dissection; ROC, receiver operating characteristics; TIMP, tissue inhibitor of metalloproteinase.

\section{DISCUSSION}

The present study had the following results: 1) Mean values of serum MMP-2, serum TIMP-1, and serum TIMP-2 differed significantly in unfavorable $v s$ favorable AAD; 2) Multivariate analysis demonstrated that values of serum TIMP-1 and serum TIMP-2 in the acute phase were independently related to outcomes in AAD; 3) A TIMP1/TIMP-2 ratio cut-off value $>3.34$ provided reliable predictive powers with a sensitivity of $88.2 \%$, specificity of
$80.0 \%$, positive predictive value of $53.6 \%$, and negative predictive value of $96.3 \%$; 4) In patients stratified according to maximum aortic dissection diameter, a TIMP-1/TIMP-2 ratio $\geq 3.34$ provided additional confirmation of a poor outcome in AAD patients who had a maximum aortic dissection diameter in excess of $46.8 \mathrm{~mm}$.

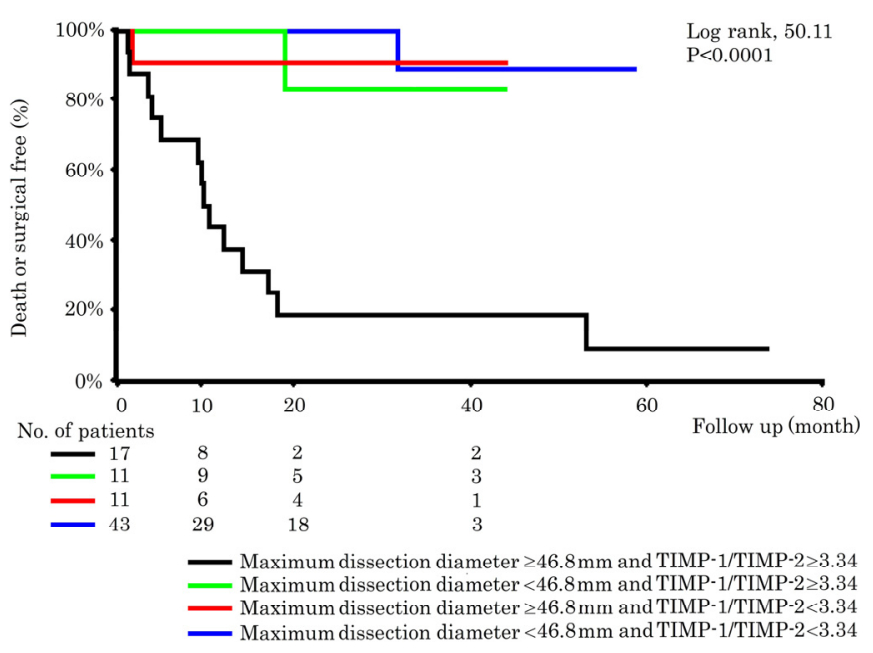

Fig. (2). Kaplan-Meier survival curve - Unadjusted Kaplan-Meier survival curve for time to endpoint including death or surgical repair of aorta in AAD patients stratified by maximum aortic dissection diameter ( $<$ or $\geq 46.8 \mathrm{~mm}$ ) and the TIMP-1/TIMP-2 ratio $(<$ or $\geq 3.34)$. AAD, acute aortic dissection; ROC, receiver operating characteristics; TIMP, tissue inhibitor of metalloproteinase.

Dissecting aneurysms of the thoracic aorta develop as a result of progressive weakening of the aortic wall. Inflammation may contribute to degeneration of the aortic wall during the development of aortic dissections. The pathologic features of AAD include degeneration of the medial elastic fibers, thinning of the media, loss of smooth muscle cells, adventitial hypertrophy, and accumulation of lymphocytes and macrophages secreting several kinds of enzymes, particularly MMPs which have been implicated in medial degradation $[10,13,14]$.

MMP-2 and MMP-9 degrade type IV collagen and elastin $[15,16]$. The activity of MMPs is regulated by TIMPs. TIMPs comprise a family of four protease inhibitors: TIMP-1, TIMP-2, TIMP-3, and TIMP-4. They are small $(\sim 23 \mathrm{kDa})$, cysteine-rich proteins with a large N-terminal domain and a smaller $\mathrm{C}$ terminal domain [17]. Reportedly, increased MMP-9 and MMP-2 expression in macrophages was observed at the site of the intimal tear in 6 patients with atherosclerosis related dissections $[12,18]$. Several clinical studies have shown an increase in MMP-9 levels in the acute and subacute phase of both type A and B aortic dissection [19, 20]. A catabolic action by MMP-9 on elastin proteins may result in weakening of the mechanical properties of the aorta, predisposing it to expansion and/or rupture [21]. MMP-2 also may be involved in the degeneration of aortic tissue [22]. In our study, AAD patients who had either a favorable or unfavorable outcome showed higher levels of MMP-9 than normal $(38 \pm 15 \mathrm{ng} / \mathrm{ml})$, in accordance with previous studies. Although no significant differences were observed in multivariate analysis, univariate analysis demonstrated an unfavorable outcome in AAD patients with higher level of MMP-2. 
Table 4. Univariate Cox Regression Analysis of MDD and TIMP-1/TIMP-2 Ratio

\begin{tabular}{|c|c|c|}
\hline Variable & HR (95\% CI) & $\mathbf{p}$ \\
\hline \multicolumn{3}{|c|}{ Versus control (MDD $<46.8 \mathrm{~mm}$ and TIMP-1/TIMP-2<3.34) } \\
\hline MDD $<46.8 \mathrm{~mm}$ and TIMP-1/TIMP-2 $\geq 3.34$ & $2.7(0.17-43.11)$ & 0.489 \\
\hline MDD $\geq 46.8 \mathrm{~mm}$ and TIMP-1/TIMP-2 $<3.34$ & $4.2(0.26-67.17)$ & 0.311 \\
\hline MDD $\geq 46.8 \mathrm{~mm}$ and TIMP-1/TIMP- $2 \geq 3.34$ & $45.6(5.96-348.42)$ & $<0.001$ \\
\hline
\end{tabular}

CI, confidence interval; HR, hazard ratio; MDD, maximum dissection diameter; TIMP, tissue inhibitor of metalloproteinase: Data are presented as mean \pm SD unless otherwise indicated. Significant $\mathrm{P}$ values $(<0.05)$ by analysis of variance followed by univariate Cox regression analysis are expressed in the bold face.

In parallel with changes in MMP-2 and MMP-9, multivariate analysis indicated that a higher TIMP-1 level, a lower TIMP-2 level, and a higher TIMP-1/TIMP-2 ratio were significant and independent risk factors for an unfavorable outcome of AAD. The degradation of collagen and elastin caused by MMPs and TIMPs produced by medial smooth muscle cells may lead to medial dissection or an intimal tear [12]. However, it is not clear whether changes in TIMP levels occur exclusively in response to increased expression of MMP-2 and MMP-9 resulting from progressive destruction of the aortic wall. TIMP-1 acts against all members of the collagenase, stromelysin, and gelatinase class of enzymes [23], and TIMP-2 is known in particular to inhibit MMP-2 [24].

The increase in proteolytic activity that may be responsible for AAD is not necessarily reflected in TIMP expression [19]. Our findings suggest that TIMP-2 was expressed at a low level and MMP-2 at a high level, compatible with the view that the former change favors matrix degradation. However, the elevation of MMP-2 might also favor expansion or rupture. In addition to the elevation of MMP-2 and MMP-9, there was a significant elevation of TIMP-1 in AAD patients with an unfavorable $v s$ a favorable outcome, suggesting either a causal role for this change, or the possibility that it represents an epiphenomenon related to upregulation of MMP-2 and MMP-9 in association with inflammatory matrix destruction in human aortic aneurysms $[21,25,26]$. The significant difference in TIMP-1 between the favorable and unfavorable outcome groups in our study might be attributable at least in part to the changes observed in MMP activity [27]. In contrast, a study by Manabe et al. reported that MMP-2 and TIMP-2 were significantly lower in aortic samples from patients with acute aortic dissection than in controls [28]. The precise mechanism of imbalance of MMPs and TIMPs and the clinical significance of the observed changes remains to be elucidated.

Mortality in AAD remains high even with successful initial hospitalization and effective medical treatment carried into the chronic phase $[2,3]$. The poor prognosis in AAD emphasizes the need for correct and timely decisions about treatment and proper staging of these patients. Besides the well recognized determinants of prognosis in AAD, i.e. aneurysm diameter, female gender, older age, cardiac tamponade, partial thrombosis of false lumen, renal failure, and hypotension/shock [29], additional factors may be of importance in identifying aortic dissection at high risk of rupture and progression. Our observation that measurement of the TIMP-1/TIMP-2 ratio is directly proportional to outcomes in AAD patients suggests the usefulness of this parameter for risk stratification. TIMP-1 and TIMP-2 may have an important clinical application for discriminating unfavorable $v s$ favorable results in AAD patients.

In our study, maximum dissection diameter was significantly greater by multivariate analysis in patients with an unfavorable $v s$ a favorable outcome, and the measurement had predictive power potential. Patients with AAD were also stratified according to TIMP-1/TIMP-2 ratio and maximum dissection diameter in our study. As a result, we found by Kaplan-Meier analysis that values in excess of 3.34 for the TIMP-1/TIMP-2 ratio and over $46.8 \mathrm{~mm}$ for maximum dissection diameter characterized a group that had a significantly reduced survival compared to other groups. This result might have an additional potential as an effective predictor of long-term prognosis in AAD.

Several potential limitations may impact the data analysis of this study, as with other observational studies: (i) As there were no good prior studies reported, sample size could not be estimated accurately; (ii) This was a retrospective study of consecutive patients, and the period over which data was collected was arbitrarily selected; (iii) There are many still undefined mechanisms with regard to the effects of TIMP-1 and TIMP-2 in AAD, and further investigation is required; (iv) The increased levels of the TIMP-1/TIMP-2 ratio in aortic dissection were not compared with histopathological examinations. Although these limitations warrant further investigation, high values of TIMP-1, low values of TIMP-2, and an increase in the TIMP-1/TIMP-2 ratio in the present study may provide an important determinant for vulnerability which may have the potential of providing insights into the progression and rupture of aortic dissection.

\section{CONCLUSIONS}

Maximum dissection diameter, high TIMP-1 and low TIMP-2 were significantly associated with an increased risk for death or surgical repair in AAD patients. Stratification of AAD patients based on the TIMP-1/TIMP-2 ratio during the acute phase of AAD may therefore be of importance in predicting long-term outcomes, and facilitate effective management of AAD.

\section{ACKNOWLEDGMENTS}

In addition to the authors, the following researchers in our hospital participated in the study: Toshiki Kawamiya, and Takeshi Hibino. We are indebted to Norio Sugimoto, MSc, for review and support with the statistical analysis. We also thank the nurses and laboratory staff in our hospital for their contributions. Funding for this observational study was provided by the Gifu Prefectural Government National Health Care Fund (Gifu Prefecture, Japan). 


\section{CONFLICT OF INTEREST}

Declared none.

\section{REFERENCES}

[1] Chiesa R, Melissano G, Civilini E, de Moura ML, Carozzo A, Zangrillo A. Ten years experience of thoracic and thoracoabdominal aortic aneurysm surgical repair: lessons learned. Ann Vasc Surg 2004; 18: 514-20.

[2] Nienaber CA, Eagle KA. Aortic dissection: new frontiers in diagnosis and management: Part I: from etiology to diagnostic strategies. Circulation 2003; 108: 628-35.

[3] Suzuki T, Mehta RH, Ince H, et al. Clinical profiles and outcomes of acute type B aortic dissection in the current era: lessons from the International Registry of Aortic Dissection (IRAD). Circulation 2003; 108: 312-7.

[4] Estrera A, Miller C, Lee TY, et al. Acute type A intramural hematoma: analysis of current management strategy. Circulation 2009; 120: 287-91.

[5] Kitai T, Kaji S, Yamamuro A, et al. Clinical outcomes of medical therapy and timely operation in initially diagnosed type A aortic intramural hematoma: a 20-year experience. Circulation 2009; 120 : 292-8.

[6] Song JK, Yim JH, Ahn JM, et al. Outcomes of patients with acute type A aortic intramural hematoma. Circulation 2009; 120: 204652 .

[7] Olsson C, Thelin S, Stahle E, Ekbom A, Granath F. Thoracic aortic aneurysm and dissection: increasing prevalence and improved outcomes reported in a nationwide population-based study of more than 14,000 cases from 1987 to 2002. Circulation 2006; 114: 26118 .

[8] Song JM, Kim SD, Kim JH, et al. Long-term predictors of descending aorta aneurysmal change in patients with aortic dissection. J Am Coll Cardiol 2007; 50: 799-804

[9] Reeps C, Essler M, Pelisek J, Seidl S, Eckstein HH, Krause BJ. Increased 18F-fluorodeoxyglucose uptake in abdominal aortic aneurysms in positron emission/computed tomography is associated with inflammation, aortic wall instability, and acute symptoms. J Vasc Surg 2008; 48: 417-23; discussion 424.

[10] Gong Y, Hart E, Shchurin A, Hoover PJ. Inflammatory macrophage migration requires MMP-9 activation by plasminogen in mice. J Clin Invest 2008; 118: 3012-24.

[11] Segura AM, Luna RE, Horiba K, et al. Immunohistochemistry of matrix metalloproteinases and their inhibitors in thoracic aortic aneurysms and aortic valves of patients with Marfan's syndrome. Circulation 1998; 98(Suppl. 19): II331-7; discussion II337-8.

[12] Ishii T, Asuwa N. Collagen and elastin degradation by matrix metalloproteinases and tissue inhibitors of matrix metalloproteinase in aortic dissection. Hum Pathol 2000; 31: 640-6.

[13] Lopez CA, Holmes DR, Liao S, Scott MJ, Wickline SA, Thompson RW. Decreased vascular smooth muscle cell density in medial degeneration of human abdominal aortic aneurysms. Am J Pathol 1997; 150: 993-1007.
[14] Tahara N, Kai H, Ishibashi M, et al. Simvastatin attenuates plaque inflammation: evaluation by fluorodeoxyglucose positron emission tomography. J Am Coll Cardiol 2006; 48: 1825-31.

[15] Dollery CM, McEwan JR, Henney AM. Matrix metalloproteinases and cardiovascular disease. Circ Res 1995; 77: 863-8.

[16] Matrisian LM. The matrix-degrading metalloproteinases. Bioessays 1992; 14(7): 455-63.

[17] Brew K, Dinakarpandian D, Nagase H. Tissue inhibitors of metalloproteinases: evolution, structure and function. Biochim Biophys Acta 2000; 1477: 267-83

[18] Tamura K, Sugisaki Y, Kumazaki T, Tanaka S. Atherosclerosisrelated aortic dissection. Kyobu Geka 2000; 53: 194-201.

[19] Sangiorgi G, Trimarchi S, Mauriello A, et al. Plasma levels of metalloproteinases- 9 and -2 in the acute and subacute phases of type $\mathrm{A}$ and type $\mathrm{B}$ aortic dissection. J Cardiovasc Med (Hagerstown) 2006; 7: 307-15.

[20] Wen T, Liu L, Xiong GZ. Matrix metalloproteinase levels in acute aortic dissection, acute pancreatitis and other abdominal pain. Emerg Med J 2009; 26: 715-8.

[21] Newman KM, Ogata Y, Malon AM, et al. Identification of matrix metalloproteinases 3 (stromelysin-1) and 9 (gelatinase B) in abdominal aortic aneurysm. Arterioscler Thromb 1994; 14: 131520 .

[22] Akiyama M, Ohtani H, Sato E, Nagura H, Tabayashi K. Upregulation of matrix metalloproteinase-2 and membrane-type 1matrix metalloproteinase were coupled with that of type I procollagen in granulation tissue response after the onset of aortic dissection. Virchows Arch 2006; 448: 811-21.

[23] Galis ZS, Sukhova GK, Lark MW, Libby P. Increased expression of matrix metalloproteinases and matrix degrading activity in vulnerable regions of human atherosclerotic plaques. J Clin Invest 1994; 94: 2493-503.

[24] Docherty AJ, Murphy G. The tissue metalloproteinase family and the inhibitor TIMP: a study using cDNAs and recombinant proteins. Ann Rheum Dis 1990; 49: 469-79.

[25] Freestone T, Turner RJ, Coady A, Higman DJ, Greenhalgh RM, Powell JT. Inflammation and matrix metalloproteinases in the enlarging abdominal aortic aneurysm. Arterioscler Thromb Vasc Biol 1995; 15: 1145-51.

[26] Herron GS, Unemori E, Wong M, Rapp JH, Hibbs MH, Stoney RJ. Connective tissue proteinases and inhibitors in abdominal aortic aneurysms. Involvement of the vasa vasorum in the pathogenesis of aortic aneurysms. Arterioscler Thromb 1991; 11: 1667-77.

[27] Zaltsman AB, George SJ, Newby AC. Increased secretion of tissue inhibitors of metalloproteinases 1 and 2 from the aortas of cholesterol fed rabbits partially counterbalances increased metalloproteinase activity. Arterioscler Thromb Vasc Biol 1999; 19: $1700-7$.

[28] Manabe T, Imoto K, Uchida K, Doi C, Takanashi Y. Decreased tissue inhibitor of metalloproteinase-2/matrix metalloproteinase ratio in the acute phase of aortic dissection. Surg Today 2004; 34 : 220-5.

[29] Tsai TT, Evangelista A, Nienaber CA, et al. Partial thrombosis of the false lumen in patients with acute type B aortic dissection. N Engl J Med 2007; 357: 349-59. 